ARTIGO ORIGINAL

\title{
Caracterização física e química de tocos e raízes de Eucalyptus para bioenergia
}

\author{
Physical and chemical characterization of Eucalyptus stumps and roots for \\ bioenergy
}

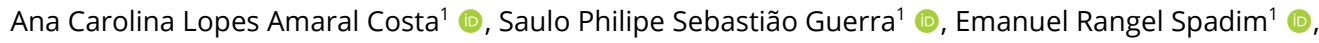
José Raimundo de Souza Passos ${ }^{1}$ (1), Humberto de Jesus Eufrade-Junior ${ }^{1}$ (1)

${ }^{1}$ Universidade Estadual Paulista - UNESP. Botucatu, SP, Brasil

Como citar: Costa, A. C. L. A., Guerra, S. P. S., Spadim, E. R., Passos, J. R. S., Eufrade-Junior, H. J. (2021).

Caracterização física e química de tocos e raízes de Eucalyptus para bioenergia. Scientia Forestalis, 49(129), e3433. https://doi.org/10.18671/scifor.v49n129.19

\begin{abstract}
Resumo
O objetivo deste trabalho foi realizar a determinação das propriedades físicas (umidade base úmidaUBU e densidade básica - DB), poder calorífico superior (PCS) e a composição química de tocos e raízes de plantações de eucalipto para diferentes clones e tempos de estocagem. Os tocos foram cavaqueados, estocados e amostrados após 0, 15, 30, 45, 60, 75 e 90 dias. Para a análise estatística das propriedades e dos fatores de variação, foram ajustados modelos lineares generalizados e os tratamentos foram comparados por meio do teste de Tukey-Kramer $(p<0,05)$. Dentre os clones estudados, Eucalyptus grandis, obteve características mais interessantes para a bioenergia (PCS de 17,1 $\mathrm{MJ} \mathrm{kg}^{-1}$, UBU de 26,2\% e menores teores de cinzas e cloro) e a estocagem não afetou a qualidade da biomassa armazenada. Os tocos têm potencial para geração de energia após colheita dos povoamentos florestais, embora novos estudos devam ser conduzidos para avaliar o uso sustentável dessa biomassa residual no Brasil.
\end{abstract}

Palavras-chave: Análise imediata; Poder calorífico; Estocagem; Cavacos.

\begin{abstract}
The objective of this work was to determine the physical properties (moisture content wet basis - UBU and basic density - DB), the higher heating value (PCS) and the chemical composition of stumps and roots from different Eucalyptus clonal plantation and storage time. The stumps were chipped, stocked and sampled after $0,15,30,45,60,75$ and 90 days. For the statistical analysis of the properties and the variation factors, generalized linear models were adjusted and the treatments were compared by the Tukey-Kramer test $(\mathrm{p}<0.05)$. Among the studied clones, Eucalyptus grandis obtained the more interesting characteristics for bioenergy (PCS of $17.1 \mathrm{MJ} \mathrm{kg-}{ }^{1}$, UBU of $26.2 \%$ and lower contents of ash and chlorine) and the storage did not affect the quality of the biomass. Stumps have the potential for power generation after harvesting from forest stands, although further studies should be conducted to evaluate the sustainable use of this residual biomass in Brazil.
\end{abstract}

Keywords: Proximate analysis, Heating value; Storage; Woodchips.

\section{INTRODUÇÃO}

O desenvolvimento econômico e tecnológico proporcionado pelo uso de combustíveis fósseis causam impactos negativos no meio ambiente (Sweidan \& Alwaked, 2016). A busca por novas formas de geração de energia limpa e sustentável é a nova tendência no desenvolvimento industrial, este tema é de interesse tanto das pequenas quanto das grandes

Fonte de financiamento: Nenhuma.

Conflito de interesse: nada a declarar

Autor correspondente: hdjejunior@gmail.com

Recebido: 26 julho 2019.

Aceito: 29 janeiro 2020.

Editor: Paulo Henrique Müller Silva.

(c) (i) Este é um artigo publicado em acesso aberto (Open Access) sob a licença Creative Commons Attribution, que permite uso, distribuição e By reprodução em qualquer meio, sem restrições desde que o trabalho original seja corretamente citado. 
economias, que buscam um alinhamento global para um crescimento econômico sustentável (Jorgenson \& Dietz, 2015).

A bioenergia é considerada uma das principais potenciais fontes de energia limpa (Bentsen \& Møller, 2017) e seu reconhecimento como uma importante alternativa para geração de energia cresce em escala global (Jorgenson \& Dietz, 2015). Tal reconhecimento é confirmado por pesquisas relacionadas ao tema, sendo os termos biomassa, bioenergia e energias renováveis os quatro mais frequentes nos últimos 20 anos dentro desta área de pesquisa (Mao et al., 2018).

O eucalipto figura entre as culturas florestais mais plantadas devido à sua alta produtividade (Binkley et al., 2017; Machado et al., 2019) e seu uso para fins energéticos (como lenha, carvão, briquetes, pellets e cavacos) já está consolidado no mercado florestal (Zanuncio et al., 2014; Eloy et al., 2016; Eufrade-Junior et al., 2016; Juizo et al., 2017).

No aproveitamento das árvores de eucalipto, os tocos e a raízes são deixados no campo após a colheita florestal, sendo arrancados e destruídos na reformulação do plantio (Gominho et al., 2012). Embora alguns autores tenham conduzido trabalhos com o uso de tocos para a energia em países europeus (Hyvönen et al., 2016; Ortiz et al., 2016; Pinto et al., 2017) faltam informações acerca da composição física e química da biomassa residual proveniente de plantações de eucalipto no Brasil.

A utilização da biomassa para fins energéticos pressupõe a determinação de algumas de suas propriedades, sendo sua composição química imediata (teores de umidade, cinzas, carbono fixo e materiais voláteis) e o poder calorífico fatores balizadores dentre estas características (Monedero et al., 2016).

Com isso, o objetivo deste trabalho foi realizar a caracterização física e química da biomassa residual (tocos e raízes) de plantações florestais de Eucalyptus spp., avaliando o efeito de clone e tempo de estocagem na qualidade da biomassa como combustível sólido.

\section{MATERIAL E MÉTODOS}

\section{Coleta do material e delineamento experimental}

A matéria-prima utilizada neste estudo foi proveniente de plantios clonais de Eucalyptus grandis $\times$ Eucalyptus camaldulensis, Eucalyptus urophylla $\times$ Eucalyptus grandis e Eucalyptus grandis, todos com sete anos de idade e conduzidos no espaçamento $3,6 \mathrm{~m} \times 2,2 \mathrm{~m}$ (1262 árvores ha-1), no município de Três Lagoas, no estado de Mato Grosso do Sul. O local é situado na longitude $51^{\circ} 40^{\prime} 42^{\prime \prime}$ W e latitude $20^{\circ} 45^{\prime} 04^{\prime \prime}$ S e possui aproximadamente $319 \mathrm{~m}$ de altitude. O clima predominante no município, segundo os critérios adotados por Koppen, é $\mathrm{cfa}$ - clima tropical - com temperaturas médias superiores a $24^{\circ} \mathrm{C}$, temperatura do mês mais quente igual ou superior a $26^{\circ} \mathrm{C}$ e índice pluviométrico anual em torno de $1241 \mathrm{~mm}$.

O fuste das árvores foi utilizado como madeira de processo, já a biomassa residual deixada no solo, caracterizada pelos tocos e raízes, foi arrancada com o auxílio de uma pácarregadora após colheita florestal semimecanizada das florestas. De cada clone foram coletados pelo menos três tocos provenientes das classes diamétricas superior, média e inferior das plantações, formando uma amostra composta por clone.

A biomassa residual foi transportada, cavaqueada, empilhada sobre um piso cimentado e armazenada no pátio de uma indústria sem cobertura ao longo do tempo. As pilhas de cavacos foram amostradas em sete ocasiões, sendo a 0,15, 30, 45, 60, 75, e 90 dias após a picagem e compreendeu de dezembro de 2016 a março de 2017.

Desta forma, o experimento foi caracterizado como um esquema fatorial $(3 \times 7)$ com dois fatores de variação, sendo três clones e sete tempos de estocagem, totalizando 21 tratamentos. 


\section{Caracterização das propriedades físicas e químicas da biomassa residual}

Para análise da qualidade da biomassa de tocos e raízes para energia foram determinadas as propriedades físicas - densidade básica e umidade, e as propriedades químicas - composição química elementar, análise imediata e poder calorífico superior. Nas análises foram realizadas três repetições para a determinação das propriedades, ambas conduzidas em duplicata. As normas técnicas nos ensaios laboratoriais estão indicadas na Tabela 1.

Tabela 1: Normas técnicas utilizadas para determinação da qualidade energética dos tocos e raízes de eucalipto.

\begin{tabular}{|c|c|c|}
\hline Propriedades & Siglas & Normas Técnicas \\
\hline Umidade & UBU & $\begin{array}{c}\text { ASTM E871 - } 82 \text { (American Society for Testing and } \\
\text { Materials, 2006a) }\end{array}$ \\
\hline Densidade básica dos cavacos & DB & $\begin{array}{c}\text { ABNT NBR } 11941 \text { (Associação Brasileira de Normas } \\
\text { Técnica, 2003) }\end{array}$ \\
\hline Poder calorífico superior & PCS & $\begin{array}{c}\text { ASTM E711 - } 87 \text { (American Society for Testing and } \\
\text { Materials, 2004) }\end{array}$ \\
\hline Carbono fixo & CF & $\begin{array}{c}\text { ASTM E870 - } 82 \text { (American Society for Testing and } \\
\text { Materials, 2006b) }\end{array}$ \\
\hline Materiais voláteis & MV & $\begin{array}{c}\text { ASTM E872 - } 82 \text { (American Society for Testing and } \\
\text { Materials, 2006c) }\end{array}$ \\
\hline Cinzas & $C Z$ & $\begin{array}{c}\text { ASTM D1102 - } 84 \text { (American Society for Testing and } \\
\text { Materials, 2007) }\end{array}$ \\
\hline $\begin{array}{l}\text { Carbono, hidrogênio, nitrogênio } \\
\text { e enxofre }\end{array}$ & CHNS & Método Pregl-Dumas \\
\hline Oxigênio & $\mathrm{O}$ & $\begin{array}{c}\text { ASTM E870-82 (American Society for Testing and } \\
\text { Materials, 2006b) }\end{array}$ \\
\hline Cloro & $\mathrm{Cl}$ & Cromatografia de íons \\
\hline
\end{tabular}

Nas análises químicas, a biomassa foi seca em estufa a $103 \pm 2^{\circ} \mathrm{C}$ até a obtenção da sua massa seca. A mesma passou pelo moinho tipo Willey e foi classificada na fração de 40-60 mesh antes dos ensaios.

Já a densidade básica da biomassa residual foi realizada com o material na forma de cavacos. Para isso, os cavacos foram saturados em água imediatamente após a coleta e a obtenção do volume saturado ocorreu pelo método da balança hidrostática.

Todas as determinações foram realizadas no Laboratório Agroflorestal de Biomassa e Bioenergia (LABB/IPBEN), localizado na Faculdade de Ciências Agronômicas (FCA/UNESP), Botucatu, no Estado de São Paulo.

\section{Análise estatística}

Para a análise estatística das variáveis UBU, DB, PCS, C, H, N e O, tendo como fatores o clone e tempo de coleta, foram ajustados modelos lineares generalizados com a distribuição Inversa Gaussiana e função de ligação logarítmica (Nelder \& Wedderburn, 1972). Já para as $\mathrm{CZ}$, MV, CF, S e Cl, foram ajustados modelos lineares generalizados com a distribuição gamma e função de ligação logarítmica (Nelder \& Wedderburn, 1972).

Foi utilizado o procedimento genmod do Programa estatístico SAS - Free Statistical Statistical Software, SAS University Edition e para comparações entre tratamentos foi utilizado foi o teste de Tukey-Kramer (Westfall et al., 1999). A qualidade dos ajustes de todos os modelos lineares generalizados foi feita por meio da análise de desvios (deviance) gráficos dos resíduos de Pearson padronizados. 


\section{RESULTADOS E DISCUSSÕES}

Nos resultados encontrados não houve efeito significativo dos fatores de variação (clone e tempo de estocagem) para o teor de nitrogênio, densidade básica dos cavacos e o poder calorífico superior da biomassa residual. Para o restante das propriedades físicas e químicas, apenas o fator de variação - clone foi significativo. A Tabela 2 apresenta as propriedades físicas da biomassa de tocos e raízes de clones de eucalipto.

Tabela 2: Valores médios das propriedades físicas dos tocos e raízes das plantações de eucalipto.

\begin{tabular}{|c|c|c|}
\hline Clone & $\begin{array}{c}\text { Umidade base úmida } \\
(\%)\end{array}$ & $\begin{array}{c}\text { Densidade básica } \\
\left(\mathrm{kg} \mathrm{m}^{-3}\right)\end{array}$ \\
\hline Eucalyptus grandis $\times$ E. camaldulensis & 25,8 B $(0,99)$ & 365 A (25) \\
\hline Eucalyptus urophylla $\times \mathrm{E}$. grandis & 36,6 A $(1,51)$ & 357 A (25) \\
\hline Eucalyptus grandis & 26,2 B $(2,38)$ & 359 A (21) \\
\hline
\end{tabular}

*Médias seguidas de mesmas letras maiúscula não diferem estatisticamente pelo teste de Tukey-Kramer ao nível de 95\% de confiança. Erro padrão da média entre parêntesis.

Os teores de umidade da biomassa residual variaram de 25 a 37\% (base úmida) e está dentro da faixa praticada na queima direta de cavacos em caldeiras industriais, sendo que a maior umidade foi encontrada para o clone de Eucalyptus urophylla $\times$ E. grandis.

A baixa densidade básica encontrada no material pode estar relacionada a presença das raízes no material cavaqueado, como reportado por Freitas et al. (2019), que estudaram os tocos e raízes de plantações florestais de eucalipto e pinus. Os mesmos autores dividiram a biomassa residual em: tocos (biomassa residual acima do solo após a colheita florestal), coroa da raiz (biomassa imediatamente abaixo do solo) e as raízes (grossas e finas), e concluíram que os tocos e a coroa da raiz são as frações de biomassa residual que poderiam ser utilizadas para energia desde que fosse feita uma análise ambiental minuciosa do impacto da extração dos tocos no solo.

O tempo de estocagem não possuiu efeito significativo em nenhuma das propriedades analisadas. Para as propriedades químicas, tal fato pode ser associado ao curto período de tempo monitorado de 0 a 90 dias. Já para a umidade da biomassa, o armazenamento de cavacos em pilhas descobertas contribuiu para manutenção da umidade ao longo do tempo, assim como constatado por Eufrade Junior et al. (2016) para pilhas de cavacos provenientes do fuste e da copa de sistemas florestais de curta rotação de eucalipto, esses autores ainda reportaram que as melhores condições de secagem dos cavacos acontecem em ambientes cobertos e com revolvimento da pilha.

Na Tabela 3 estão apresentados teores dos elementos químicos da biomassa residual de eucalipto. De fato, os valores não diferem dos que foram encontrados por Gominho et al. (2012) que avaliaram tocos de Eucalyptus globulus aos 24 anos em Portugal e são similares à madeira de Eucalyptus grandis x E. urophylla (Zanuncio et al., 2019).

Tabela 3: Valores médios da composição química elementar de tocos de diferentes clones de Eucalyptus spp.

\begin{tabular}{ccccccc}
\hline Clone & $\mathbf{C ~ ( \% )}$ & $\mathbf{H ~ ( \% )}$ & $\mathbf{O}(\%)$ & $\mathbf{N}(\%)$ & $\mathbf{S ~ ( \% )}$ & $\mathbf{C l}(\%)$ \\
\hline \multirow{2}{*}{ Eucalyptus grandis $\times$ camaldulensis } & $46,08 \mathrm{C}$ & $4,99 \mathrm{C}$ & $41,55 \mathrm{~A}$ & $0,32 \mathrm{~A}$ & $0,063 \mathrm{~A}$ & $0,478 \mathrm{~B}$ \\
& $(0,96)$ & $(0,11)$ & $(1,74)$ & $(0,03)$ & $(0,00)$ & $(1,03)$ \\
Eucalyptus urophylla $\times$ grandis & $46,24 \mathrm{~B}$ & $5,10 \mathrm{~B}$ & $39,83 \mathrm{C}$ & $0,31 \mathrm{~A}$ & $0,061 \mathrm{~B}$ & $0,569 \mathrm{~A}$ \\
& $(0,46)$ & $(0,07)$ & $(1,47)$ & $(0,04$ & $(0,00)$ & $(1,29)$ \\
Eucalyptus grandis & $49,11 \mathrm{~A}$ & $5,29 \mathrm{~A}$ & $40,35 \mathrm{~B}$ & $0,29 \mathrm{~A}$ & $0,060 \mathrm{C}$ & $0,410 \mathrm{C}$ \\
& $(0,50)$ & $(0,02)$ & $(0,63)$ & $(0,05)$ & $(0,00)$ & $(0,46)$ \\
\hline
\end{tabular}

*Médias seguidas de mesmas letras maiúscula não diferem estatisticamente pelo teste de Tukey-Kramer ao nível de $95 \%$ de confiança. Erro padrão da média entre parêntesis. 
Dentre os elementos químicos, o teor de cloro é limitante na exportação desse material para outros países. Segundo as normas internacionais europeias, como por exemplo, a norma DIN 51731(Deutsches Institut Für Normung, 1996) exige que o teor de cloro médio na madeira seja menor que $0,02 \%$, este valor é muito abaixo do que o encontrado no presente trabalho (0,48\%). Também, Leonello et al. (2019) encontraram um valor médio de cloro de 0,13\% para tocos de clones de E. urophylla, E. urophylla $\times$ E. camaldulensis e E. urophylla $\times$ E. grandis, de 6 a 14 anos de idade, plantados no espaçamento $3 \mathrm{~m}$ x 3m em Ribas do Rio Pardo (MS).

Na Tabela 4 estão os resultados encontrados para análise imediata e poder calorífico superior.

Tabela 4: Valores médios da análise imediata e poder calorífico superior da biomassa de toco e raízes de eucalipto.

\begin{tabular}{ccccc}
\hline Clone & Cinzas (\%) & $\begin{array}{c}\text { Mat. voláteis } \\
\mathbf{( \% )}\end{array}$ & $\begin{array}{c}\text { Carbono Fixo } \\
\mathbf{( \% )}\end{array}$ & $\begin{array}{c}\text { PCS } \\
\mathbf{( M J ~ k g}^{-1} \mathbf{)}\end{array}$ \\
\hline $\begin{array}{c}\text { Eucalyptus grandis } \times \\
\text { camaldulensis }\end{array}$ & $2,21 \mathrm{~B}(0,31)$ & $79,04 \mathrm{~B}(0,70)$ & $18,75 \mathrm{~A}(0,54)$ & $16,9 \mathrm{~A}(1,98)$ \\
$\begin{array}{c}\text { Eucalyptus urophylla } \times \text { grandis } \\
\text { Eucalyptus grandis }\end{array}$ & $2,75 \mathrm{~A}(0,37)$ & $82,44 \mathrm{~A}(0,62)$ & $14,88 \mathrm{C}(0,51)$ & $16,6 \mathrm{~A}(1,43)$ \\
\hline $0,79 \mathrm{C}(0,04)$ & $82,36 \mathrm{~A}(0,02)$ & $16,84 \mathrm{~B}(0,37)$ & $17,1 \mathrm{~A}(1,83)$ \\
\hline
\end{tabular}

*Médias seguidas de mesmas letras maiúscula não diferem estatisticamente pelo teste de Tukey-Kramer ao nível de 95\% de confiança. Erro padrão da média entre parêntesis.

Os clones não apresentaram diferenças significativas no PCS, embora tenham ocorrido diferenças na composição química elementar dos tocos, como no teor de hidrogênio. Considerando o poder calorífico, os tocos de ambas as espécies têm potencial para gerar o mesmo conteúdo energético por massa seca, com um valor médio de $16,9 \mathrm{MJ} \mathrm{kg}^{-1}$.

Para a análise imediata, todas as propriedades variaram entre os clones. Destaque para o elevado teor de cinzas quando comparado à madeira de eucalipto, cujo valor médio é igual ou menor que 0,6\% (Juizo et al., 2017; Zanuncio et al., 2019). O teor médio de cinzas dos tocos e raízes entre os clones estudados foi de 1,92\%. Este valor pode estar ligado a contaminação pelo solo durante a operação de arranquio no campo. O Eucalyptus grandis foi aquele que obteve o menor teor de $\mathrm{CZ}$, o qual pode reduzir a quantidade de resíduos na caldeira após a queima. Sabe-se que as cinzas têm um comportamento abrasivo nas paredes metálicas dos equipamentos, além de formar incrustações (Monti et al., 2008).

De forma geral, os tocos aparecem como uma oportunidade para geração de energia, entretanto, seu uso está condicionado a novos estudos, que devem avaliar o balanço de nutrientes e de carbono no ecossistema florestal, em busca do uso sustentável dessa biomassa no Brasil.

\section{CONCLUSÃO}

A biomassa oriunda de tocos e raízes dos clones de Eucalyptus spp. tem potencial para geração de energia após colheita dos povoamentos florestais. Dentre os clones estudados, destaque para Eucalyptus grandis, que obteve um poder calorífico e densidade básica igual aos demais clones, mas com menores teores de cinzas e cloro na biomassa. A estocagem não afetou a qualidade da biomassa residual armazenada no pátio em até 90 dias após a picagem.

\section{AGRADECIMENTOS}

Os autores agradecem à Coordenação de Aperfeiçoamento de Pessoal de Nível Superior (CAPES), ao Conselho Nacional de Desenvolvimento Científico e Tecnológico (CNPq), à Faculdade de Ciências Agronômicas (FCA/UNESP), ao Instituto de Biomassa e Bioenergia (IPBEN) e ao Laboratório Agroflorestal de Biomassa e Bioenergia (LABB). 


\section{REFERÊNCIAS}

American Society for Testing and Materials - ASTM. (2004). ASTM E711-87: Standard test methods for gross calorific value of refuse-derived fuel by the bomb calorimeter (8 p.). West Conshohocken, PA: ASTM.

American Society for Testing and Materials - ASTM. (2006a). ASTM E871-82: Standard test methods for moisture analysis of particulate wood fuels (2 p.) West Conshohocken, PA: ASTM.

American Society for Testing and Materials - ASTM. (2006b). ASTM E870-82: Standard test methods for analysis of wood fuel (2 p.). West Conshohocken, PA: ASTM.

American Society for Testing and Materials - ASTM. (2006c). ASTM E872-82: Standard test methods for volatile matter in the analysis of particulate wood (3 p.). West Conshohocken, PA: ASTM.

American Society for Testing and Materials - ASTM. (2007). ASTM D1102-84: Standard test methods for ash in wood (2 p.). West Conshohocken, PA: ASTM.

Associação Brasileira de Normas Técnica - ABNT. (2003). NBR 11941: Madeira - determinação da densidade básica (6 p). Rio de Janeiro: ABNT.

Bentsen, N. S., \& Møller, I. M. (2017). Solar energy conserved in biomass: sustainable bioenergy use and reduction of land use change. Renewable \& Sustainable Energy Reviews, 71, 954-958. http://dx.doi.org/10.1016/j.rser.2016.12.124.

Binkley, D., Campo, O. C., Alvares, C., Carneiro, R. L., Cegatta, I., \& Stape, J. L. (2017). The interactions of climate, spacing and genetics on clonal Eucalyptus plantations across Brazil and Uruguay. Forest Ecology and Management, 405, 271-283.

Deutsches Institut Für Normung - DIN. (1996). DIN 51731: Testing of solid fuels - compressed untreated wood, Requirements and testing. Berlin.

Eloy, E., Da Silva, D. A., Schmidt, D., Trevisan, R., Caron, B. O., \& Elli, E. F. (2016). Efeito da idade e do espaçamento de plantio nas propriedades energéticas do eucalyptus grandis w. hill ex maiden. Revista Árvore, 40(4), 749-758. http://dx.doi.org/10.1590/0100-67622016000400019.

Eufrade-Junior, H. J., Oguri, G., Melo, R. X., Ballarin, A. W., \& Guerra, S. P. S. (2016). Storage of whole-tree chips from high-density energy plantations of Eucalyptus in Brazil. Biomass and Bioenergy, 93, 279283. http://dx.doi.org/10.1016/j.biombioe.2016.07.009.

Freitas, J. M., Ramos, J. H., Eufrade-Junior, H. D., Azevedo, G. B. A., \& Guerra, S. P. S. (2019). Histórico e detalhes do projeto de P\&D ANEEL. In S. P. S. Guerra \& H. J. Eufrade-Junior. Recuperação energética da biomassa de tocos e raízes de florestas plantadas (p. 29-37). Botucatu: FEPAF.

Gominho, J., Lourenço, A., Miranda, I., \& Pereira, H. (2012). Chemical and fuel properties of stumps biomass from Eucalyptus globulus plantations. Industrial Crops and Products, 39(1), 12-16. http://dx.doi.org/10.1016/j.indcrop.2012.01.026.

Hyvönen, R., Kaarakka, L., Leppälammi-Kujansuu, J., Olsson, B. A., Palviainen, M., Vegerfors-Persson, B., \& Helmisaari, H. S. (2016). Effects of stump harvesting on soil C and N stocks and vegetation 8-13 years after clear-cutting. Forest Ecology and Management, 371, 23-32. http://dx.doi.org/10.1016/j.foreco.2016.02.002.

Jorgenson, A. K., \& Dietz, T. (2015). Economic growth does not reduce the ecological intensity of human well-being. Sustainability Science, 10(1), 149-156. http://dx.doi.org/10.1007/s11625-014-0264-6.

Juizo, C. G. F., Lima, M. R., \& Silva, D. A. (2017). Qualidade da casca e da madeira de nove espécies de Eucalipto para produção de carvão vegetal. Agrária, 12(3), 386-390.

Leonello, E. C., Arruda, N. L. F. V., Passos, J. R. S., \& Prosdocini, A. B. P. (2019). Histórico e detalhes do projeto de P\&D ANEEL. In S. P. S. Guerra \& H. J. Eufrade-Junior. Recuperação energética da biomassa de tocos e raízes de florestas plantadas (pp. 107-122). Botucatu: FEPAF.

Machado, F. C., Eufrade-Junior, H. J., Spada, G., Oguri, G., Garcia, É. A., Guerra, S. P. S. (2019). Produção de biomassa de Eucalyptus grandis Hill ex. Maiden em diferentes arranjos de espaçamentos de plantio simples e duplos. Ciência Florestal, 29(4), 1568-1578.

Mao, G., Huang, N., Chen, L., \& Wang, H. (2018). Research on biomass energy and environment from the past to the future: A bibliometric analysis. The Science of the Total Environment, 635, 1081-1090. PMid:29710563. http://dx.doi.org/10.1016/j.scitotenv.2018.04.173.

Monedero, E., Hernández, J. J., Cañellas, I., Otero, J. M., \& Sixto, H. (2016). Thermochemical and physical evaluation of poplar genotypes as short rotation forestry crops for energy use. Energy Conversion and Management, 129, 131-139. http://dx.doi.org/10.1016/j.enconman.2016.10.021.

Monti, A., Virgílio, N., \& Venturi, G. (2008). Mineral composition and ash content of six major energy crops. Biomass and Bioenergy, 32(3), 216-223. http://dx.doi.org/10.1016/j.biombioe.2007.09.012. 
Nelder, J. A., \& Wedderburn, R. W. M. (1972). Generalized linear models. Journal of the Royal Statistical Society. Series A (General), 135(3), 370-384. http://dx.doi.org/10.2307/2344614.

Ortiz, C. A., Hammar, T., Ahlgren, S., Hansson, P. A., \& Stendahl, J. (2016). Time-dependent global warming impact of tree stump bioenergy in Sweden. Forest Ecology and Management, 371, 5-14. http://dx.doi.org/10.1016/j.foreco.2016.02.014.

Pinto, F., Gominho, J., André, R. N., Gonçalves, D., Miranda, M., Varela, F., Neves, D., Santos, J., Lourenço, A., \& Pereira, H. (2017). Improvement of gasification performance of Eucalyptus globulus stumps with torrefaction and densification pre-treatments. Fuel, 206, 289-299. http://dx.doi.org/10.1016/j.fuel.2017.06.008.

Sweidan, O. D., \& Alwaked, A. A. (2016). Economic development and the energy intensity of human wellbeing: evidence from the GCC countries. Renewable \& Sustainable Energy Reviews, 55, 1363-1369. http://dx.doi.org/10.1016/j.rser.2015.06.001.

Westfall, P. H., Tobias, R. D., Rom, D., Wolfinger, R. D., \& Hochberg, Y. (1999). Multiple Comparisons and Multiple Tests Using the SAS System (p. 416). Cary, NC: SAS Institute Inc.

Zanuncio, A. J. V., Carvalho, A. G., Carneiro, A. C. O., Colodette, J. L., \& Rocha, M. F. V. (2019). Chemical and energetic characterization of Eucalyptus grandis $\times$ Eucalyptus urophylla clones subject to wind damage. Revista Árvore, 43(4), e430401. http://dx.doi.org/10.1590/1806-90882019000400001.

Zanuncio, A. J. V., Lima, J. T., Monteiro, T. C., Trugilho, P. F., \& Lima, F. S. (2014). Secagem ao ar livre da madeira para produção de carvão vegetal. Floresta e Ambiente, 21(3), 401-408. http://dx.doi.org/10.1590/2179-8087.028713.

Contribuição dos Autores: ACLAC: investigação, escrita - primeira redação; SPSG: conceituação, administração do projeto, recursos, supervisão, escrita - revisão e edição; ERS: metodologia, escrita - revisão e edição; JRSP: curadoria dos dados, software, visualização, validação, escrita - revisão e edição; HJEJr.: conceituação, metodologia, curadoria dos dados, supervisão, escrita - revisão e edição. 